\title{
Greenhouses: A Modelica Library for the Simulation of Greenhouse Climate and Energy Systems
}

\author{
Queralt Altes-Buch $^{1 *} \quad$ Sylvain Quoilin ${ }^{1,2} \quad$ Vincent Lemort $^{1}$ \\ ${ }^{1}$ Energy Systems Research Unit - Thermodynamics Laboratory, Aerospace and Mechanical Engineering Department, \\ University of Liege, Liege, Belgium \\ ${ }^{2}$ Smart Thermal Energy Systems, KU Leuven, Geel, Belgium \\ *Corresponding author: qaltes@uliege.be
}

\begin{abstract}
This paper presents the results of an on-going project to develop Greenhouses, an open Modelica library for the simulation of greenhouse climate and energy systems. The Greenhouses library is one of the few open-source modeling frameworks for greenhouse climate and crop growth simulation, and the first able to handle simulating the energy integration of greenhouses coupled to thermal systems (e.g. generation and storage units). The proposed modeling framework can be used for multiple purposes, such as the optimal control of the greenhouse actuators, the optimal sizing of the heating appliances, or the optimal integration of the units in the power system. The Greenhouses library also comprises multiple example models, making it readily usable for both research and industrial applications.
\end{abstract}

Keywords: Greenhouse climate, CHP, Crop yield, Thermal systems, Climate Control, Dynamic modeling

\section{Introduction}

Greenhouses present the peculiarity of requiring heating, electricity and $\mathrm{CO}_{2}$. As an energy consumer, they contribute to the depletion of non-renewable energy sources and to global warming through energy-related emissions (e.g. $\mathrm{CO}_{2}$ emissions from fossil fuel combustion gases). Their energy sources should therefore provide the combined demands in a competitive but also sustainable way. Up to now, the use of combined heat and power (CHP) is proposed as an efficient technology for that purpose: the CHP thermal generation is used for heating purposes, the electricity covers the consumption of the appliances and the $\mathrm{CO}_{2}$ from the exhaust gases can be recovered to activate photosynthesis. In most cases, there is an excess electricity generation that is fed back to the grid. CHP units in greenhouse horticulture are highly flexible, with the ability to go to full load in less than one hour (Buck et al., 2014). Therefore, when coupled to thermal storage, CHP units can be valuable for the power system by providing services such as load balancing, ancillary services or decentralised storage capacity (Jiménez-Navarro et al., 2018). For example, in a country like the Netherlands, the CHP units dedicated to greenhouse horticulture produced $7.8 \%$ of the national production in 2016 (cbs, 2018). Greenhouses can also be coupled to district heatings, in which case activities such as heat recovery from the industry are made possible.

To evaluate the potential of such activities, the complex energy flows within greenhouses must be understood, which also requires ad hoc greenhouse climate models. In addition, a platform for dynamic simulation of the thermal flows interacting between greenhouses and external thermal systems (e.g. district heating networks, generation units, thermal storage) is required. In the current literature, a small number of models are openly available for grenhouse climate simulation and crop growth. Although researchers openly present model structures and simulation scenarios, an open-source simulation platform is still lacking. In fact, the most common climate simulation softwares (e.g. CASTA, KASPRO, VirtualGreenhouse) are not open-access and are not able to handle the integration of greenhouses with external thermal systems. The Greenhouses Modelica library aims at filling this gap by providing an open-source modeling framework capable of simulating greenhouse climate as well as its complex interactions with thermal systems. To that end, the library proposes models covering the following aspects:

- Greenhouse climate, to compute the energy consumption of a greenhouse given its specific design, outdoor conditions and a specific control.

- Thermal systems, with models ranging from heat distribution systems in greenhouses to generation and thermal storage units.

- Crop yield, to account for crop requirements as well as crop behavior (e.g. transpiration and photosynthesis), which influence the indoor climate and thus, the greenhouse energy consumption.

Climate control systems (heating, ventilation, $\mathrm{CO}_{2}$ enrichment and supplementary lighting) are also included in the library. Furthermore, several numerical methods are developed and implemented in order to enhance the robustness and the simulation speed of the models during initialization and integration.

The library is simple to implement and intuitive to use. The required information for a new user to get started is provided in this paper. Moreover, an additional documen- 
tation including a user guide with the required steps to run the models and extended documentation of the library content is available online (cfr. Section 5 for more details).

The library shows potential for both research and industry applications. On the one hand, it can be useful for greenhouse operators when it comes to optimizing the control of the actuators or the sizing of the HVAC appliances. On the other hand, it can be used for purposes such as optimizing the integration of CHPs in the electricity markets.

\section{The Greenhouses Modelica library}

The Greenhouses Modelica library aims at providing a robust framework to simulate greenhouse climate and its integration with energy systems. The goal is to provide an integrated and fully open-source solution ranging from the computation of energy flows in a greenhouse, to the simulation of complex systems with their control strategy. The Modelica language is thus well adapted to the formulation of this problem, mainly because of its acausal characteristic language that allows inter-connecting the models in a 'physical' way (Casella et al., 2007). The key features of the library are the following:

- Designed for system level simulations.

- Full compatibility (connector-wise) with the Modelica Standard Library and libraries such as ThermoCycle, ThermoPower or Buildings.

- Various numerical robustness strategies implemented in the components and accessible through Boolean parameters.

- High readability of the models (limited levels of hierarchical modeling).

The components provided in the library are designed to be as generic as possible. For example, the detailed geometry records of the greenhouse structure are not compulsory. Instead, only the floor area, the mean greenhouse height and the roof tilt are required. In all the models, default values relative to the most commonly used greenhouse structure (e.g. the Venlo greenhouse) are proposed for all the parameters.

\subsection{Modeling of greenhouse climate}

Greenhouse climate models have been the object of a substantial literature. While many models have been developed (Bot, 1983; De Zwart, 1996; Impron et al., 2007; Luo et al., 2005; van Ooteghem, 2010), most of them can only be used for a single location and for a specific greenhouse structure and climate. Recently, a more generic greenhouse climate model combining the work of Bot (1983) and De Zwart (1996) was developed. For the purpose of this work, this model, which was developed by Vanthoor et al. (2011b) and validated for a range of climates and greenhouse designs, has been implemented.

The model describes the indoor climate of a greenhouse resulting from the greenhouse design, the outdoor climate and a specific climate control. The indoor climate is characterized by the air temperature, water vapor pressure (to account for the air relative humidity) and $\mathrm{CO}_{2}$ concentration. Besides, the variables with an indirect influence on the climate are also modeled. These are mainly the characteristics relative to the canopy and the envelope (i.e. the cover, the floor and the thermal screen). In order to compute the indoor climate, the modeling approach consists in applying the energy conservation principles on each greenhouse component and the mass balance on the air. To that end, all the existent energy and mass flows must be modeled. A detailed description of the latter can be found in Altes-Buch and Lemort (2018). Using the encapsulation capabilities of the Modelica language, the balances and flows are defined in independent models that should be inter-connected to build the greenhouse system. The Modelica language offers a high degree of flexibility to the user because:

(i) the greenhouse structure and energy systems are not predefined, i.e. the model can easily be adapted to match different types of greenhouses

(ii) the models are parametrizable i.e. the user can define the materials and system sizes.

The main models of the library are described in the following sections. For a full description of the equations of the models, please consult the online documentation of the library in https://greenhouses-library. readthedocs.io. An example of greenhouse model is shown in Figure 1. As it can be distinguished, the greenhouse modeled in this example consists of a twolevel heating circuit, roof windows (no side vents), natural ventilation (no forced ventilation) and a movable thermal screen. It should be noted that, when the screen is drawn, the air of the greenhouse is divided in two zones, i.e. below and above the screen. These zones are modeled separately and their respective climate is assumed to be homogeneous.

\subsubsection{Surfaces}

This section describes the modeling approach used to model the cover, the floor, the canopy and the thermal screen. The energy balance on these surfaces is defined by equation (1):

$$
\rho c V \frac{d T}{d t}=\sum \dot{Q}+\sum \dot{Q}_{L}+P_{\text {Sun }}+P_{\text {Light }}
$$

which takes into account the following exchanges:

- Sensible heat flows $(\dot{Q})$, including convection with the indoor or outdoor air, long-wave radiation between all surfaces or to the sky, and conduction through the soil.

- Latent heat flows $\left(\dot{Q}_{L}\right)$, such as the heat exchanged by condensation on the inner side of the cover, condensation or evaporation on the screen, or evaporation on the leaves. These flows can be treated as forced flows, since they are determined by the mois- 


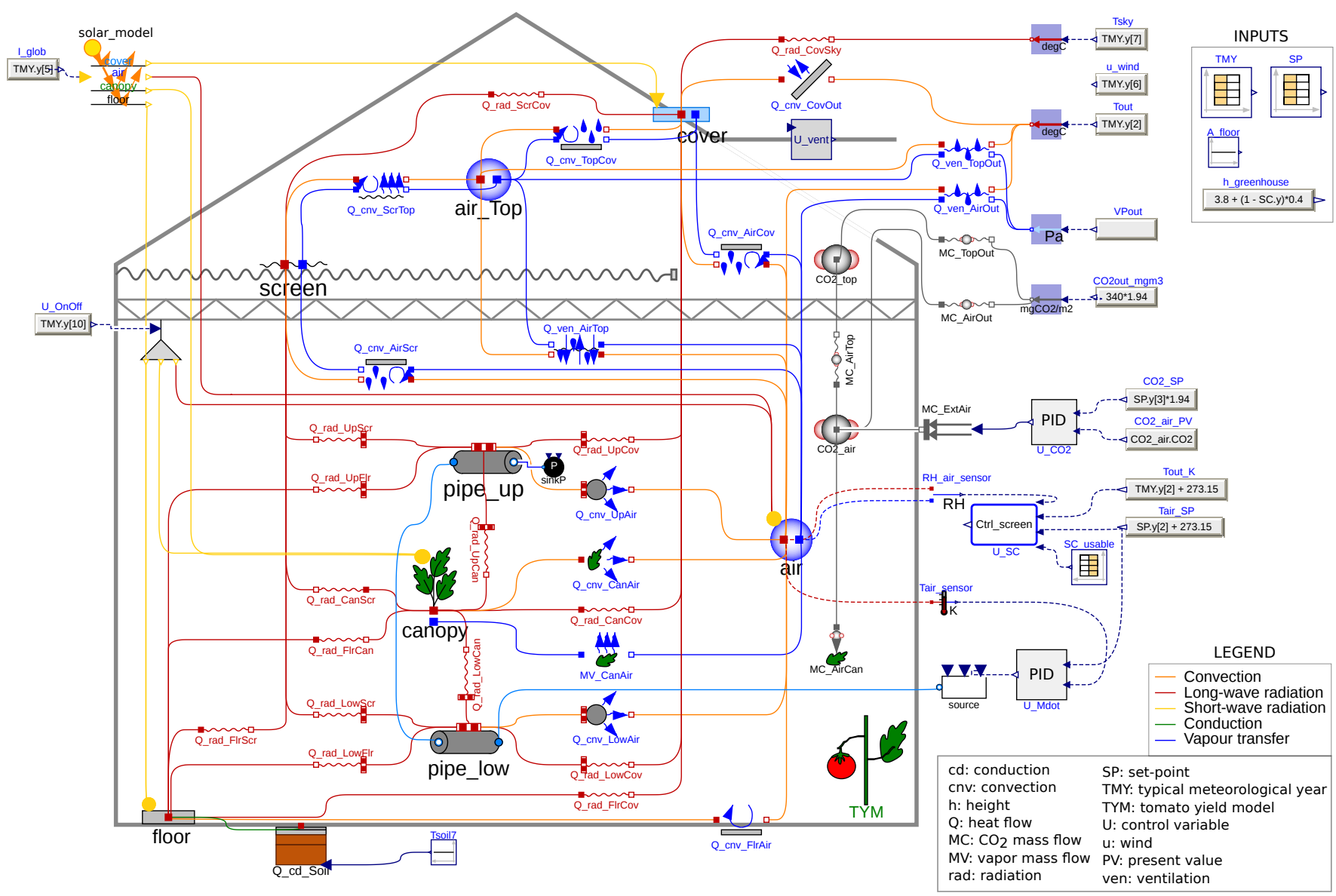

Figure 1. Graphical interface of the greenhouse climate simulation model (Greenhousel in the Examples package)

ture mass flow rate caused by condensation or evaporation $\left(\dot{M}_{v}\right)$ and the heat of evaporation $\left(\Delta h_{f g}\right)$ :

$$
\dot{Q}_{L, 12}=\Delta h_{f g} \cdot \dot{M}_{v, 12}
$$

- Short-wave radiation inputs, such as the absorbed radiation from the sun $\left(P_{S u n}\right)$ and/or supplementary lighting $\left(P_{\text {Light }}\right)$.

The water vapor pressure at a surface is defined as the saturated vapor pressure at the surface temperature. No mass balance is applied on the modeled surfaces.

\section{Cover}

The cover is the only surface exchanging with both the inside and outside air. The model (cover in Figure 1) can be parametrized for any type of glazing (single-glass, doubleglass, polycarbonate, etc.). For single glazing, since glass thickness is commonly small $(4 \mathrm{~mm})$, conduction is neglected. Depending on the vapor pressure difference, condensation may take place at the inner side of the cover. Evaporation of moisture from the cover to the air is neglected since the condensate is commonly drained.

\section{Canopy}

The magnitude of the energy exchanged by the canopy depends on the size of the leaves, which is increased with crop growth and decreased by leaf pruining. To take this into account, the leaf area index (LAI), defined as the leaf area per unit of ground area, is used. The LAI is computed in the crop yield model and input in the canopy model (canopy in Figure 1). The heat capacity per unit of leaf area is the main parameter. The canopy temperature has an impact on its photosynthesis and transpiration, which decrease the $\mathrm{CO}_{2}$ concentration and increase the moisture content of the air, respectively.

\section{Floor}

The floor model (floor in Figure 1) can be parametrized for a range of floor materials (e.g. soil, concrete). Conduction through the soil is modeled by a nodal model, dividing it into several layers. The temperature of the deepest layer is a boundary condition. Vapor transfer is not modeled.

\section{Thermal screen}

The thermal screen (waved line in Figure 1) is a membrane used to reduce the energy requirement to heat the greenhouse. When drawn, thermal losses to the outside are reduced by 38 to $60 \%$, depending on the nature of its material (Bailey, 1988). The screen model (screen in Figure 1) can easily be parametrized to cover the wide variety of commercial screens nowadays used by horticul- 
ture growers. The screen thickness, commonly less than 1 $\mathrm{mm}$, implies a very low heat capacity. Since the screen is mostly drawn at night (i.e. when there is no sunlight), the absorbed heat from short-wave radiation is neglected.

Given the porous nature of the screen, air and moisture are exchanged through its fabric. The present model assumes that the thermal screen is capable of transporting water from the lower side to the upper side. The storage of moisture in the screen is however neglected. This implies that the vapor that condenses at the screen is either evaporated at the upper side or drips from the screen. The rate of evaporation is therefore lower or equal to the rate of condensation.

\subsubsection{Air}

The energy balance on the indoor air (air in Figure 1) is defined by equation (3):

$$
\rho c_{p} V \frac{d T}{d t}=\sum \dot{Q}+P_{S u n}+P_{L i g h t}
$$

which takes into account the following exchanges:

- Sensible heat flows $(\dot{Q})$, including convection at surfaces and ventilation flows (natural, forced or leakage) with the outside air.

- Forced heat inputs, including the short-wave radiation from the sun $\left(P_{S u n}\right)$ or supplementary lighting $\left(P_{\text {Light }}\right)$, which are first absorbed by the greenhouse construction elements and later released to the air.

The moisture content of the air is increased by the transpiration of the canopy and decreased by ventilation and by condensation on the cover and the screen. In the model, it is characterized by the water vapor pressure of the air $\left(P_{v}\right)$, which is determined by the vapor mass balance defined in equation (4):

$$
M_{H} \frac{V}{R T} \frac{d P_{v}}{d t}=\sum \dot{M}_{v}
$$

where $M_{H}$ is the molar mass of vapor and $\dot{M}_{v}$ is the vapor mass flow rate.

The $\mathrm{CO}_{2}$ concentration of the greenhouse air, being independent from the heat and vapor exchanges, is computed in a separate model (CO2_air in Figure 1). Its value is decreased by ventilation processes and by the $\mathrm{CO}_{2}$ consumption of the canopy, and increased by the $\mathrm{CO}_{2}$ supply from an external source controlled by the climate controller. The $\mathrm{CO}_{2}$ mass concentration $\left(\gamma_{\mathrm{CO} 2}[\mathrm{mg}\{\mathrm{CO} 2\}\right.$ $\mathrm{m}^{-3}\{$ air $\left.\left.\}\right]\right)$ of the air is determined in the $\mathrm{CO}_{2}$ mass balance, defined in equation (5).

$$
V \frac{d \gamma_{C O 2}}{d t}=\sum \dot{M}_{c}
$$

where $\dot{M}_{c}$ is the $\mathrm{CO}_{2}$ mass flow rate.

The top air zone has a very low heat capacity and is only modeled when the screen is drawn (i.e. mostly at night, to mitigate losses in the lack of sunlight). For this reason, its heat and vapor balances are computed in a simplified version of the air model (air_Top in Figure 1), in which the heat input from short-wave radiation $\left(P_{S u n}\right.$ in equation (3)) is neglected. The $\mathrm{CO}_{2}$ balance (CO2_top in Figure 1) is done in the same manner as for the main zone.

\subsubsection{Heating pipes}

The fluid in the heating pipes from the greenhouse heating ciruit is modeled by means of the discretized model for incompressible flow described in Section 2.4. Heat is transferred by long-wave radiation to the canopy, floor and cover, and by convection to the air. Since the thermal resistance from the outer pipe surface to the air is about 100 times greater than the thermal resistance from the inner surface to the outer one (De Zwart, 1996), the temperature of the pipe surface can be assumed equal to the water temperature.

Greenhouse heating circuits are commonly made of several parallel heating loops. The main parameters of the model (pipe_low in Figure 1) are the pipe diameter, the installed length per unit of ground area per loop, and the number of parallel loops. The nominal mass flow rate and the number of nodes in which each loop is discretized are also parameters of the model.

\subsection{Modeling of heat flows}

Several models are proposed for computing the different types of heat transfer. It should be noted that convection and long-wave radiation are modeled separately.

\subsubsection{Free convection at surfaces}

The upward or downward heat exchange by free convection from an horizontal or inclined surface is modeled. The heat exchange coefficients are modeled based on the Nuselt-Rayleigh (Nu-Ra) relation (Balemans, 1989). The model can be used for convection at the cover (upward flow, inclined surface), the floor (upward/downward flow, horizontal surface) or the screen (upward flow, horizontal surface). The bi-direction nature of the convective flow on the floor is due to the fact that the latter can be warmer or colder than the air above it. The different natures of the flows lead to different $\mathrm{Nu}$-Ra relations for each surface. Therefore, the user should indicate (by means of the Boolean parameters) which surface is being modeled.

Depending on the status of the thermal screen, the heat flow to the cover can originate either from the top or the main air zone, and the heat flow to the screen can have a different magnitude. Therefore, when the model is used for the cover or the screen, the screen closure (control variable in the global system) is a required input.

\subsubsection{Free convection at the leaves}

The heat exchange coefficient on the leaves of tomato crop was derived experimentally by Stanghellini (1987). Because of the lack of required input data to compute it, in the present model it is however simplified to a constant value. This coefficient is expressed per unit of leaf area. 
In order to compute the global heat exchange coefficient, the LAI is thus a required input.

\subsubsection{Free convection at heating pipes}

The magnitude of convective heat from the heating pipes to the air depends on the pipe position, which implies a free exchange (i.e. pipes in free air) or a hindered exchange (i.e. pipes situated close to the canopy and near the floor). The free exchange is modeled based on the $\mathrm{Nu}$-Ra relation. The hindered exchange, considered to be forced, is modeled by experimental correlations derived by Bot (1983). The user should indicate which exchange should be modeled by means of a Boolean parameter. The diameter of the pipes and the installed pipe length per unit of ground area are also required parameters.

\subsubsection{Forced convection with the outside air}

The convection at the outer side of the greenhouse cover is modeled according to the experimental work of Bot (1983), who characterised the heat exchange coefficient at this saw-tooth surface as a function of the wind speed. The wind speed is an exogenous input of the model. The main parameter is the cover tilt.

\subsubsection{Natural ventilation}

The heat transfer between the inside and outside air due to natural ventilation is computed as a function of the air exchange rate. This rate, derived by Boulard and Baille (1993), depends mainly on two factors. The first one is the window opening, a required input which is set by the climate controller. The second one is the window characteristics (e.g. the wind pressure coefficient and the coefficient of energy discharge caused by friction at the windows), which in order to simplify the model, are set to constant values relative to standard roof windows.

Depending on the status of the thermal screen, the heat flow can originate either from the top or the main air zones. Therefore, the screen closure (control variable from the climate controller) is also a required input.

This model also takes into account the leakage rate through the greenhouse structure, which is dependent on the wind speed (exogenous input of the model) and the leakage coefficient of the greenhouse (parameter of the model, characteristic of its structure).

\subsubsection{Forced ventilation}

The heat flow from forced ventilation is computed as a function of the air exchange rate between two air volumes, which depends on the capacity of the ventilation system (parameter of the model) and the position of the control valve (required input set by the climate controller).

\subsubsection{Ventilation through the screen}

Analogously to the other ventilation models, the heat transfer caused by air exchange between the main and top air zones is computed as a function of the air exchange rate, which is the sum of the air rates caused by two mechanisms. The first one is the air exchange through the openings in the fabric of the screen, which is temperature driven and was derived experimentally by Balemans (1989). The second one is the exchange through the gap when the screen is opened, which is caused by density difference and was theoretically modeled by Miguel (1998) using the Navier-Stokes equation. The main required input is the screen closure (control variable from the climate controller).

\subsubsection{Long-wave radiation}

The long-wave infrared radiation flows are modeled for each exchange between all the surfaces in the greenhouse (red lines in Figure 1). These flows are modeled by the Stefan-Boltzmann equation. The emission coefficients, characteristic of the surfaces, are parameters of the model for which a standard value is proposed in the documentation of the model. The view factor of each surface is computed according to De Zwart (1996) in its component model and is an input of the model.

\subsubsection{Short-wave radiation}

Short-wave radiation in a greenhouse can be originated from the sun or from supplementary lighting.

\section{Solar model}

The main input is the solar radiation incident in a greenhouse, which can be split in three spectral parts: ultra violet (UV, from 0.3 to $0.4 \mu \mathrm{m}$ ), visible light (from 0.4 to $0.7 \mu \mathrm{m}$ ) and near infrared light (NIR, from 0.7 to $3 \mu \mathrm{m}$ ). The visible light has an interest for biological growth and is referred as photosynthetically active radiation (PAR) in greenhouse modeling. The fraction of UV and PAR in the global radiation is 6-10\% and $45-60 \%$, respectively (Coulson, 1975). However, for plant growth it is common to assign 50\% to PAR, neglect the UV and assign the other $50 \%$ to NIR (De Zwart, 1996). Besides the spectral division, the solar radiation can be divided in direct and diffuse radiation. The solar model of this work is simplified by making no distinction between diffuse and direct solar radiation and by assuming that the transmission coefficient of the greenhouse cover does not depend on the solar angle. It should be remarked that the optical properties of the greenhouse elements differ for PAR and NIR.

On the cover, the incident radiation from the sun is partially reflected, absorbed and transmitted inside the greenhouse. The transmitted radiation is absorbed by the construction elements, the canopy or the floor. The transmitted PAR to be absorbed by the canopy or the floor is defined by:

$$
\dot{q}_{P A R, \tau}=\left(1-\eta_{G l o b, A i r}\right) \cdot \tau_{C o v, P A R} \cdot \eta_{G l o b, P A R} \cdot I_{G l o b}
$$

where $\eta_{G l o b, A i r}$ is the ratio of the radiation that is absorbed by the greenhouse construction elements, $\tau_{C o v, P A R}$ is the transmission coefficient of the cover and $\eta_{G l o b, P A R}$ is the fraction of PAR in the outside global radiation $\left(I_{G l o b}\right)$. When the thermal screen is closed, $\tau_{C O v, P A R}$ is a lumped transmission coefficient of the greenhouse cover and the movable thermal screen. 
For instance, the PAR absorbed by the canopy is the sum of the PAR transmitted by the cover and directly absorbed by the canopy and the PAR reflected by the floor and later absorbed by the canopy. In a homogenous crop, this is described by an exponential decomposition of light with the LAI (Ross, 1975):

$$
\begin{array}{r}
\dot{q}_{P A R, C a n}=\dot{q}_{P A R, \tau}\left(1-\rho_{C a n, P A R}\right)\left(1-e^{-K_{P A R} \cdot L A I}\right)+ \\
\dot{q}_{P A R, \tau} \cdot e^{-K_{P A R} \cdot L A I} \cdot \rho_{F l r, P A R}\left(1-\rho_{C a n, P A R}\right)\left(1-e^{-K_{P A R} \cdot L A I}\right)
\end{array}
$$

where $\rho_{C a n, P A R}$ and $\rho_{F l r, P A R}$ are the reflection coefficients for PAR of the canopy and the floor, and $K_{P A R}$ is the extinction coefficient for PAR of the canopy.

\section{Supplementary lighting}

Although the contribution of supplementary lighting is very small during summer, in winter it can double the sun input during a day and thus, have an important impact on crop growth. The illumination model is designed for high intensity discharge lamps (e.g. high pressure sodium (HPS) lamps) and the main parameter is the installed power per unit of ground area. For these lamps, only $17 \%$ and $25 \%$ of the electrical power is converted into NIR and PAR, respectively. The remaining $58 \%$ is released to the greenhouse air (Urban and Urban, 2010). The fraction of radiation absorbed by the greenhouse components is computed similarly than in the solar model.

\subsection{Modeling of moisture and $\mathrm{CO}_{2}$ flows}

This section presents the modeling approach for the computation of moisture and $\mathrm{CO}_{2}$ flows.

\subsubsection{Condensation and evaporation}

The mass exchange coefficients for condensation and evaporation at the screen and the cover are linearly related to their convective heat exchange coefficients by a conversion factor (De Zwart, 1996). As previously stated, evaporation from the cover and from the screen's lower side is not modeled. Therefore, the mass flow rates due to condensation are prohibited from being negative. Condensation on the upper side of the screen is prohibited as well. Negative flows are avoided by setting the mass transfer coefficients to zero when the water vapor pressure difference between the air and the surface is negative.

\subsubsection{Mass transfer through ventilation}

Mass transfer occurs in ventilation processes, i.e. between the main and top air zones, and between these and the outside air. The moisture and $\mathrm{CO}_{2}$ flows accompanying an air exchange are function of the air flow rate, which is computed as explained in Sections 2.2.5 and 2.2.7.

\subsubsection{Mass transfer at the canopy}

The canopy transpiration originates from a phase interface somewhere inside the cavities of a leaf. The resistance to moisture transport from the leaves to the air was derived by Stanghellini (1987) as a function of leaf temperature,
$\mathrm{CO}_{2}$ concentration of the air, water vapor pressure difference and absorbed solar irradiation. These variables, computed elsewhere, are inputs of this sub-model. Furthermore, transpiration is also function of the dimension of the leaves. The LAI is therefore an input of the model.

The $\mathrm{CO}_{2}$ flow from the air absorbed by the canopy depends on the canopy photosynthesis rate and the respiration processes. It is computed in the crop yield model and input in this model.

\subsection{Modeling of fluid flows}

Fluid flows are modeled using the finite volume approach by means of a discretized model for incompressible flow, adapted from Quoilin et al. (2014). The model distinguishes between two types of variables: cell and node variables. The main features and hypothesis of the model can be summarized by:

- Dynamic energy balance and static mass and momentum balance are applied in each cell

- Upwind or central differences discretization scheme

- Uniform velocity through the cross section and constant pressure

- Axial thermal energy transfer is neglected

The overall flow model can be built by connecting several cells in series. The model is compatible with the $\mathrm{Me}$ dia package of the Modelica Standard Library, at the condition that the considered fluid is incompressible.

\subsection{Modeling of HVAC systems}

In the Greenhouses library, several HVAC models are provided in order to enable system-level simulations such as the energy integration of greenhouses with generation and storage units. To that end, performance-based models of CHP units, heat pump and thermal storage units are developed. Although the number of modeled HVAC systems remains limited, the full compatibility (connector-wise) of the Greenhouses library allows the connection with other libraries more specialized in modeling thermal systems (e.g. Buildings, ThermoCycle, ThermoPower, etc.). In all the developed HVAC models, fluid flow is modeled by means of the fluid model described in Section 2.4. To illustrate the modeling possibility of the Greenhouses library, two system-level simulations are included in the $E x$ amples package.

\subsubsection{CHP}

The CHP model does not consider part-load operation (ON/OFF regulation is assumed). Thus, constant natural gas consumption and total efficiency are assumed. The electrical efficiency is computed assuming a constant second-law efficiency, whose value is obtained using the nominal operating conditions.

\subsubsection{Heat pump}

For heat pumps, two models are proposed. First, a peformance-based model similar to the CHP model is de- 
veloped, in which the second-law efficiency is assumed to remain unchanged in part-load operation.

A second more detailed model is also implemented, in which the heat pump performance are predicted at both full- and partial-load operation by three polynomial laws fitted through manufacturing data (Bolther et al., 1999).

\subsubsection{Thermal energy storage}

The thermal energy storage model is a nodal model of a stratified tank with an internal heat exchanger and ambient heat losses, adapted from Quoilin et al. (2014). The water tank is modeled using the energy and mass conservation principles and assuming thermodynamic equilibrium at all times inside the control volume. The following hypothesis are applied:

- No heat transfer between the different nodes.

- The internal heat exchanger is discretized in the same way as the tank: each cell of the heat exchanger corresponds to one cell of the tank and exchanges heat with that cell only.

- Incompressible fluid in both the tank and the heat exchanger.

- Axial thermal conductivity is neglected.

\subsection{Modeling of crop yield}

Several inputs used in the computation of the greenhouse climate (e.g. the LAI, the $\mathrm{CO}_{2}$ flow absorbed by the canopy) are characteristics of the crop and should be quantified by a crop growth model. Moreover, with a crop growth model, the yield and hence, the profitability (e.g. savings in energy) from different control strategies can be compared. For those reasons, a dynamic crop yield model is implemented. Given that yield models differ between crops, the model implemented in this work is only valid for tomato crop.

Crop growth is related to photosynthesis and most of the existent crop yield models directly relate these two variables without considering a carbohydrate buffer. The buffer is a storage system of the crop, whose function is to store the carbohydrates from the photosynthesis (inflow) before they are distributed to the plant organs (outflow). It has a maximum capacity, above which carbohydrates cannot be stored anymore, and a lower limit, below which the carbohydrate outflow stops. Thus, the in- and out-flows depend on the level of carbohydrates in the buffer and thereby, may not be simultaneous. For instance, crop growth may continue after dusk, when photosynthesis has stopped but distribution can still be possible if the buffer content has not yet reached its lower limit. The presence of a carbohydrate buffer is thus important when modeling crop growth, as suggested in Dayan et al. (1993); Heuvelink (1996); Linker et al. (2004); Marcelis et al. (1998); Seginer et al. (1994).

In this work, a recent yield model developed and validated for a variety of temperatures (Vanthoor et al., 2011a) is implemented. The model structure is shown in Figure 2.
The carbohydrate assimilation is modeled by distinguishing three crop parts: the leaves, the fruits and the stems (and roots). Mass balances are applied on each part and on the buffer. For instance, the mass balance on the buffer is described by:

$$
\begin{array}{r}
\frac{d C_{B u f}}{d t}=\dot{M}_{C, \text { AirBuf }}-\dot{M}_{C, B u f F r u i t}-\dot{M}_{C, B u f L e a f}- \\
\dot{M}_{C, B u f S t e m}-\dot{M}_{C, B u f A i r}
\end{array}
$$

where $C_{B u f}$ is the availability of carbohydrates in the buffer and $\dot{M}_{C}$ are the carbohydrate flows, which are computed as a function of fixed parameters related to the tomato crop. The inputs of the model are the instantaneous temperature of the canopy, the $\mathrm{CO}_{2}$ concentration of the greenhouse air and the PAR absorbed by the canopy. Their values are retrieved from the greenhouse climate simulation model. The main outputs of the model are the LAI, the harvested dry matter, the photosynthesis rate and the respiration rates.

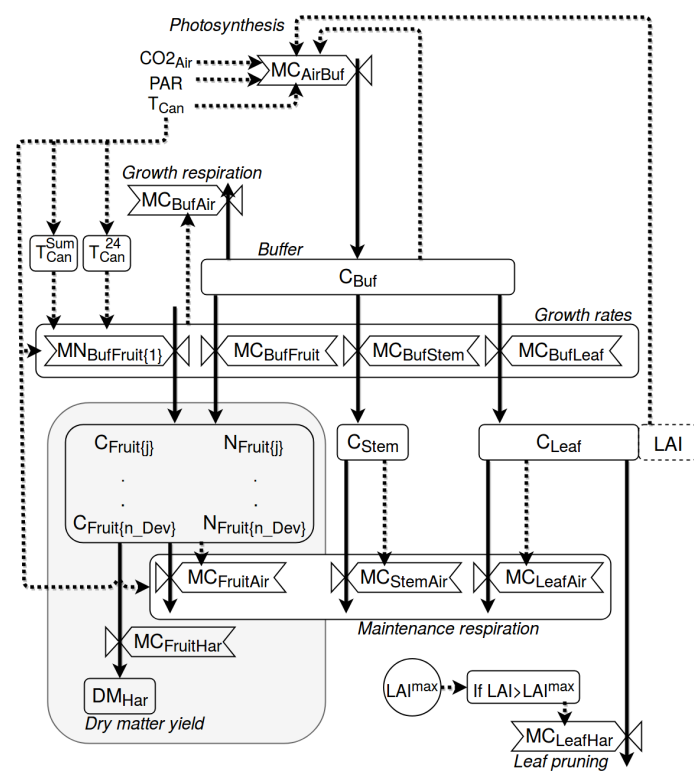

Figure 2. Schematic representation of the crop yield model. Boxes define state variables (blocks), semi-state variables (dotted blocks) and carbohydrate flows (valves). Arrows define mass flows (solid lines) and information flows (dotted lines). Adapted from Vanthoor et al. (2011a).

\section{Numerical aspects}

The complexity of the final model largely depends on the selected discretization scheme for the piping and for the ground. However, for a typical complete greenhouse example model (e.g. the model Greenhousel in the Examples package), the system of equations comprises 4222 unknowns, among which 197 are differentiated variables. After the symbolic manipulation, the size of the non-linear systems of equations is 236 for the initialization problem and 3 for the integration. The typical solving time is 48 minutes for a one-year simulation with a $3 \mathrm{GHz}$ I7 processor. 
Because of the important time constants involved in some parts of the model (e.g. the vapor content of the air within the greenhouse), most equations are initialized in steady-state. While this adds some complexity to the initialization problem (in the current example, a system of 236 non-linear equations), it avoids long and unnecessary transients at the beginning of the simulation.

Some equations of the model include conditional statements (in the form of equation (9)) which, during integration, generate state events and therefore decrease the computational efficiency of the model (Jorissen et al., 2015).

$$
y= \begin{cases}y_{1} & \text { if } k>k_{s} \\ y_{2} & \text { otherwise }\end{cases}
$$

In order to increase the computational efficiency of the model, these conditional statements have been replaced by a differentiable switch function. For the general case where $y_{1}, y_{2} \in \mathbb{R}$, the statement is replaced by:

$$
y=y_{1} \cdot S_{k}+y_{2} \cdot\left(1-S_{k}\right)
$$

where $S_{k}$ is the value of a differentiable switch function that is determined by the state variable $k$, which is defined by:

$$
S_{k}=\frac{1}{1+e^{s_{k}\left(k-k_{s}\right)}}
$$

where $k_{s}$ is the value of $k$ where $S_{k}$ is 0.5 , and $s$ is the slope of the differentiable switch at $k_{s}$. The sign of $s$ is set according to if $S_{k}$ increases $(s<0)$ or decreases $(s>$ 0 ) with an increasing $k$. For instance, in the case where some crop parameters differ between day and night, $k$ is the global irradiation, $k_{s}$ is equal to zero, and $y_{1}$ and $y_{2}$ are the values of the parameter at daytime and nighttime, respectively.

The model also includes conditional statements in which the output value is equal to the indicator function, defined by equation (12).

$$
y= \begin{cases}1 & \text { if } k \in\left[k_{s 1}, k_{s 2}\right] \\ 0 & \text { otherwise }\end{cases}
$$

These conditional statements are approximated by:

$$
y=S_{k}^{1} \cdot S_{k}^{2}
$$

where $S_{k}^{1}$ and $S_{k}^{2}$ are two differentiable switch functions, which are defined according to equation (11) for $k_{s 1}$ and $k_{s 2}$ and have opposite slope signs (i.e. the former is negative, the latter is positive).

\section{Library implementation}

\subsection{Library structure}

The Greenhouses library is hierarchically structured into different packages, including:

- Components, is the central part of the library. It is organized in three sub-packages:

- Greenhouse, contains models from the simple greenhouse components (i.e. all the models described in Section 2.1) to already-build greenhouse models ready to use (similar to Figure 1);
- HVAC, contains the models for generation and storage units presented in Section 2.5;

- CropYield, contains the yield model for tomato crop described in Section 2.6.

- Flows, contains models of the flows that are encountered in a greenhouse system. It is organized in seven sub-packages that model the heat, moisture and $\mathrm{CO}_{2}$ mass transfer, as well as fluid flow. These models are described from Section 2.2 to 2.4 .

- ControlSystems, organized in two sub-packages, contains control units to control Climate (i.e. the thermal screen closure, the operation of supplementary lighting and the window's aperture) and HVAC (i.e. the operation of generation units, the storage (dis-)charge) (cfr. Section 4.2 for more details).

- Examples, contains examples that demonstrate the usage of this library. It includes simulations of greenhouses (e.g. Figure 1) and two system-scale simulations of a greenhouse connected to a thermal storage, a CHP and a heat pump (e.g. Figure 4).

- Interfaces, contains all the type of connectors used in the library.

- Functions, contains the empirical correlations used to characterize some of the models presents in the library.

Figure 3 shows an overview of the library structure.

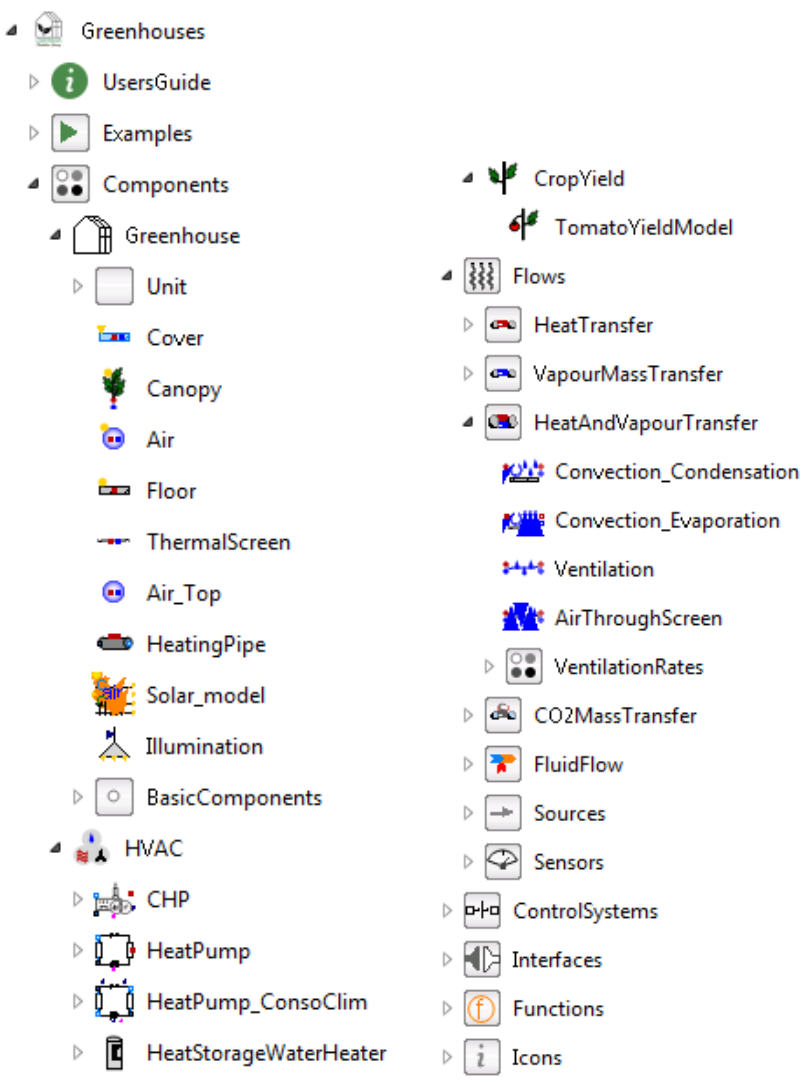

Figure 3. An overview of the library structure from the Dymola graphical user interface 


\subsection{Control Systems}

Greenhouses have high requirements on indoor climate control. The control strategies used in commercial climate controllers differ from manufacturers and are commonly private-access. For this reason, several control strategies for the control of climate systems are developed. The implemented control strategies are based on a literature review on climate requirements and control practices (Aaslyng et al., 2003; Bailey, 1988; De Zwart, 1996; Dieleman and Kempkes, 2006; Grange and Hand, 1987; Grisey and Brajeul, 2007; Urban and Urban, 2010; Vanthoor et al., 2011a). In the library, depending on the nature of the strategies, two implementation approaches are distinguished: proportional-integral (PI) and state graph based controllers. The library includes models for the control of:

- Supplementary lighting: ON/OFF operation determined by a state graph based controller. The strategy sets up a time window for lighting, during which a lighting set-point condition is applied. To prevent cycling, natural light levels must be below or above the set-point for a proving time, and once turned on, lights must remain on for a minimum time.

- Natural ventilation: a PI controller sets the windows' aperture based on air sanitation and air cooling, i.e. the air relative humidity and temperature are not allowed to increase above a certain value.

- Thermal screen: the screen's closure is set by a state graph based controller model. The screen deployment is done progressively as a function of the outside irradiation. Depending on the night, a small temporary opening of the screen may be required to regulate humidity or temperature.

- Heating: a PI controller adjusts the heating power output by varying the supply mass flow rate of the heating pipes according to the difference between the air temperature set-point and actual value.

- $\mathrm{CO}_{2}$ external source: a PI controller adapts the $\mathrm{CO}_{2}$ supply rate to attain the set-point. In high ventilation conditions, $\mathrm{CO}_{2}$ enrichment is commonly reduced due to the high exchange rate to the outside air.

The developed control strategies remain relatively simple compared to some state-of-the-art commercial climate controllers. Users are therefore encouraged to develop their own controls systems adapted to their climate requirements.

\section{Open-source implementation}

Quality of science relies upon basic principle such as reproducibility, transparency or peer-review, which are greatly facilitated by open-source and open-data approaches (Pfenninger et al., 2017). For this reason, the presented library is released as open-source (using the permissive Modelica License 2). The required documentation for a new user to use the models is described in this paper. The library can be downloaded from https: / / github.com/queraltab/GreenhouseLibrary.

In addition to this paper, an online documentation of the library is available in https: //greenhouses-library.readthedocs.io. Apart from an overview of the library, the online documentation includes a user guide with the required steps for a new user to get started. Furthermore, it includes an extended description of each model of the library, in which the main modeling assumptions and equations are stated. To demonstrate the usage of the library, the example simulations from the Examples package are also commented.

\section{Conclusion}

The development of the Greenhouses library is an ongoing process aiming at providing a completely opensource tool for the simulation of greenhouse climate and its energy integration with thermal systems or the power system. The library comprises a number of components that can be used to simulate a wide range of greenhouse structures and climates. Moreover, the crop growth model allows determining the yield, and hence, the profitability of different control strategies. The components can finally be used to simulate the coupling of greenhouses with generation units and thermal storage, as proposed by the authors in a previous publication Altes-Buch et al. (2018) and illutrated in Figure 4. In that work the library was used to optimize the control of a greenhouse connected to a CHP, a heat pump and a storage system in such a way to maximize self-consumption, leading to significant savings ( $9 \%$ of the total operation cost) compared to the baseline.

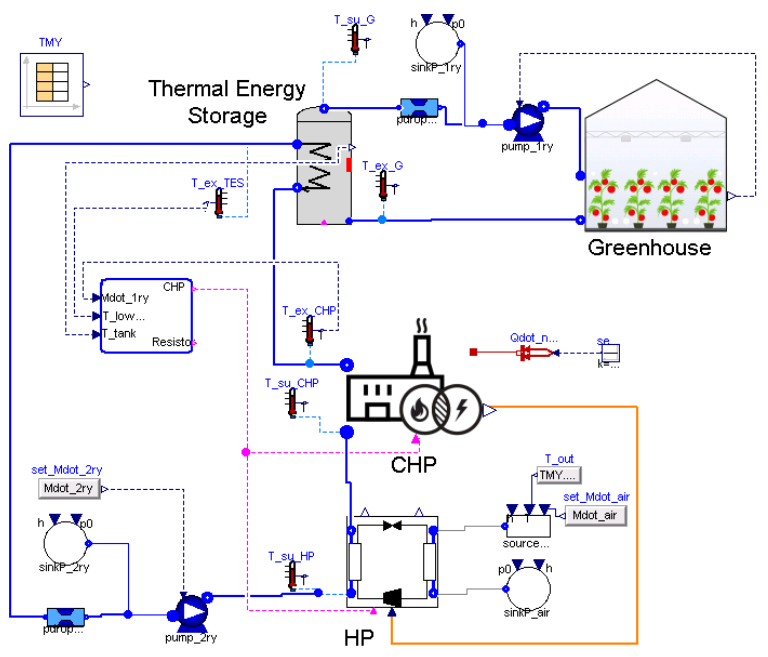

Figure 4. Diagram of a simulation example

The full compatibility (connector-wise) of the library allows the connection with other libraries more specialized in modeling thermal systems, thus increasing the simulation possibilities of the Greenhouses library. The library is released as open-source, ensuring a proper reproducibility and re-usability of this work. Ongoing and fu- 
ture works will mainly focus on the integration of new components and on the validation of the proposed models.

\section{Acknowledgements}

The authors thank the Walloon Region of Belgium, which has funded this research in the context of the EcoSystemePass project (convention 1510610).

\section{References}

CBS StatLine. Electricity; production and means of production, 2018. URL https://opendata.cbs.nl/statline. Last accessed 16 January 2018.

J. M. Aaslyng, J. B. Lund, N. Ehler, and E. Rosenqvist. IntelliGrow: a greenhouse component-based climate control system. Environmental Modelling \& Software, 18(7):657-666, September 2003. ISSN 1364-8152. doi:10.1016/S1364-8152(03)00052-5.

Q. Altes-Buch and V. Lemort. Modeling framework for the simulation and control of greenhouse climate. In Proceedings of the 10th International Conference on System Simulation in Buildings, Liege, December 2018.

Q. Altes-Buch, S. Quoilin, and V. Lemort. Modeling and control of CHP generation for greenhouse cultivation including thermal energy storage. In Proceedings of the 31st international conference on efficiency, cost, optimization, simulation and environmental impact of energy systems, Guimaraes, Portugal, June 2018.

B. J. Bailey. Control strategies to enhance the performance of greenhouse thermal screens. Journal of Agricultural Engineering Research, 40(3):187-198, July 1988. ISSN 0021-8634. doi:10.1016/0021-8634(88)90206-5.

L. Balemans. Assessment of criteria for energetic effectiveness of greenhouse screens. PhD thesis, Agricultural University, Ghent, 1989.

A. Bolther, Casari R., Fleury E., D. Marchio, and J.R. Millet. Méthode de calcul des consommations d'énergie des bâtiments climatisés ConsoClim. Technical Report CSTB ENEA/CVA-99.176R, Ecole des Mines, Paris, 1999.

G.P.A Bot. Greenhouse climate : from physical processes to a dynamic model. $\mathrm{PhD}$ thesis, Wageningen University, 1983.

T. Boulard and A. Baille. A simple greenhouse climate control model incorporating effects of ventilation and evaporative cooling. Agricultural and Forest Meteorology, 65(3):145-157, August 1993. ISSN 0168-1923. doi:10.1016/0168-1923(93)90001-X.

A. Buck, S. Hers, M. Afman, H. Croezen, F. Rooijers, W. van der Veen, P. van der Wijk, and T. Slot. The Future of Cogeneration and Heat Supply to Industry and Greenhouse Horticulture. Technical Report 14.3D38.67, CE Delft, Delft, October 2014.

F. Casella, J. G. van Putten, and P. Colonna. Dynamic Simulation of a Biomass-Fired Steam Power Plant: A Comparison Between Causal and A-Causal Modular Modeling. pages 205-216, January 2007. doi:10.1115/IMECE2007-41091.

K. L. Coulson. Solar and Terrestrial Radiation. Elsevier, 1975. ISBN 978-0-12-192950-3. doi:10.1016/B978-0-12-192950-3.X5001-3.

E. Dayan, H. van Keulen, J. W. Jones, I. Zipori, D. Shmuel, and H. Challa. Development, calibration and validation of a greenhouse tomato growth model: I. Description of the model. Agricultural Systems, 43(2):145-163, January 1993. ISSN 0308-521X. doi:10.1016/0308-521X(93)90024-V.

H.F. De Zwart. Analyzing energy-saving options in greenhouse cultivation using a simulation model. $\mathrm{PhD}$ thesis, Wageningen University, 1996.

J.A. Dieleman and F.L.K. Kempkes. Energy screens in tomato: determining the optimal opening strategy. Acta Horticulturae, (718):599-606, October 2006. ISSN 0567-7572, 2406-6168. doi:10.17660/ActaHortic.2006.718.70.

R. I. Grange and D. W. Hand. A review of the effects of atmospheric humidity on the growth of horticultural crops. Journal of Horticultural Science, 62(2):125-134, January 1987. ISSN 0022-1589. doi:10.1080/14620316.1987.11515760.
A. Grisey and E. Brajeul. Serres chauffées: réduire ses dépenses énergétiques. Centre technique interprofessionnel des fruits et légumes (CTIFL), 2007.

E. Heuvelink. Tomato growth and yield: quantitative analysis and synthesis. $\mathrm{PhD}$ thesis, Wageningen University, 1996.

I. Impron, S. Hemming, and G. P. A. Bot. Simple greenhouse climate model as a design tool for greenhouses in tropical lowland. Biosystems Engineering, 98(1):79-89, September 2007. ISSN 1537-5110. doi:10.1016/j.biosystemseng.2007.03.028.

J. P. Jiménez-Navarro, K. C. Kavvadias, S. Quoilin, and A. Zucker. The joint effect of centralised cogeneration plants and thermal storage on the efficiency and cost of the power system. Energy, 149:535-549, April 2018. ISSN 0360-5442. doi:10.1016/j.energy.2018.02.025.

F. Jorissen, M. Wetter, and L. Helsen. Simulation Speed Analysis and Improvements of Modelica Models for Building Energy Simulation. In Proceedings of the 11th International Modelica Conference, Versailles, France, September 2015. Lawrence Berkeley National Lab. (LBNL), Berkeley, CA (United States).

R. Linker, I. Seginer, and F. Buwalda. Description and calibration of a dynamic model for lettuce grown in a nitrate-limiting environment. Mathematical and Computer Modelling, 40(9):1009-1024, November 2004. ISSN 0895-7177. doi:10.1016/j.mcm.2004.12.001.

W. Luo, H.F. de Zwart, J. DaiI, X. Wang, C. Stanghellini, and C. Bu. Simulation of Greenhouse Management in the Subtropics, Part I: Model Validation and Scenario Study for the Winter Season. Biosystems Engineering, 90(3):307-318, March 2005. ISSN 1537-5110. doi:10.1016/j.biosystemseng.2004.11.008.

L. F. M Marcelis, E Heuvelink, and J Goudriaan. Modelling biomass production and yield of horticultural crops: a review. Scientia Horticulturae, 74(1):83-111, April 1998. ISSN 0304-4238. doi:10.1016/S0304-4238(98)00083-1.

A. A. F. Miguel. Transport phenomena through porous screens and openings : from theory to greenhouse practice. $\mathrm{PhD}$ thesis, Wageningen University, January 1998.

S. Pfenninger, J. DeCarolis, L. Hirth, S. Quoilin, and I. Staffell. The importance of open data and software: Is energy research lagging behind? Energy Policy, 101:211-215, February 2017. ISSN 03014215. doi:10.1016/j.enpol.2016.11.046.

S. Quoilin, A. Desideri, J. Wronski, I. Bell, and V. Lemort. ThermoCycle: A Modelica library for the simulation of thermodynamic systems. In Proceedings of the 10th International Modelica Conference 2014, 2014

J. Ross. Radiative transfer in plant communities. In Vegetation and Atmosphere (Ed. J. L. Monteith), pages 13-55. Academic Press, London, UK, 1975.

I. Seginer, C. Gary, and M. Tchamitchian. Optimal temperature regimes for a greenhouse crop with a carbohydrate pool: A modelling study. Scientia Horticulturae, 60(1):55-80, December 1994. ISSN 03044238. doi:10.1016/0304-4238(94)90062-0.

C. Stanghellini. Transpiration of greenhouse crops : an aid to climate management. $\mathrm{PhD}$ thesis, Wageningen University, 1987.

L. Urban and I. Urban. La production sous serre: La gestion du climat, volume 1. Lavoisier, 2nd edition, 2010.

R. J. C. van Ooteghem. Optimal Control Design for a Solar Greenhouse. IFAC Proceedings Volumes, 43(26):304-309, January 2010. ISSN 1474-6670. doi:10.3182/20101206-3-JP-3009.00054.

B. H. E. Vanthoor, P. H. B. de Visser, C. Stanghellini, and E. J. van Henten. A methodology for model-based greenhouse design: Part 2 , description and validation of a tomato yield model. Biosystems Engineering, 110(4):378-395, December 2011a. ISSN 1537-5110. doi:10.1016/j.biosystemseng.2011.08.005.

B. H. E. Vanthoor, C. Stanghellini, E. J. van Henten, and P. H. B. de Visser. A methodology for model-based greenhouse design: Part 1 , a greenhouse climate model for a broad range of designs and climates. Biosystems Engineering, 110(4):363-377, December 2011b. ISSN 1537-5110. doi:10.1016/j.biosystemseng.2011.06.001. 